\title{
Inequality of Ostrowski Type for Mappings with Bounded Fourth Order Partial Derivatives
}

\author{
Waseem Ghazi Alshanti (iD \\ Department of General Studies, Jubail University College, Saudi Arabia \\ Correspondence should be addressed to Waseem Ghazi Alshanti; shantiw@ucj.edu.sa
}

Received 19 October 2018; Revised 7 January 2019; Accepted 15 January 2019; Published 3 March 2019

Academic Editor: Patricia J. Y. Wong

Copyright (C) 2019 Waseem Ghazi Alshanti. This is an open access article distributed under the Creative Commons Attribution License, which permits unrestricted use, distribution, and reproduction in any medium, provided the original work is properly cited.

A general Ostrowski's type inequality for double integrals is given. We utilize function whose partial derivative of order four exists and is bounded.

\section{Introduction}

In 1938, Ostrowski [1] introduced the following integral inequality.

Theorem 1. Let $f:[a, b] \longrightarrow \mathbb{R}$ be continuous mapping on $[a, b]$ and differentiable on $(a, b)$, whose derivative $f^{\prime}$ : $(a, b) \longrightarrow \mathbb{R}$ is bounded on $(a, b)$, i.e., $\left\|f^{\prime}\right\|_{\infty}=\sup _{t \in[a, b]}\left|f^{\prime}(t)\right|$ $<\infty$, then for all $x \in[a, b]$

$$
\begin{aligned}
\mid f & (x)-\frac{1}{b-a} \int_{a}^{b} f(t) d t \mid \\
& \leq\left[\frac{1}{4}+\frac{(x-(a+b) / 2)^{2}}{(b-a)^{2}}\right](b-a)\left\|f^{\prime}\right\|_{\infty} .
\end{aligned}
$$

The constant $1 / 4$ is sharp in the sense that it cannot be replaced by a smaller one.

In 1975, Milovanović [2] proposed the following generalization of (1) for a function $f$ of several variables whose first order partial derivatives are bounded.

Theorem 2. Let $f: \mathbb{R}^{m} \longrightarrow \mathbb{R}$ be a differentiable function defined on $\bar{D}$ and let $\left|\partial f / \partial x_{i}\right| \leq M_{i}\left(M_{i}>0 ; i=1, \ldots, m\right)$ in $D$. Then, for every $X=\left(x_{1}, \ldots, x_{m}\right) \in \bar{D}$,

$$
\mid f\left(x_{1}, \ldots, x_{m}\right)
$$

$$
\begin{aligned}
& -\frac{1}{\prod_{i=1}^{m}\left(b_{i}-a_{i}\right)} \int_{a_{1}}^{b_{1}} \cdots \int_{a_{m}}^{b_{m}} f\left(y_{1}, \ldots, y_{m}\right) d y_{1} \cdots d y_{m} \\
& \leq \sum_{i=1}^{m}\left[\frac{1}{4}+\frac{\left(x_{i}-\left(a_{i}+b_{i}\right) / 2\right)^{2}}{\left(b_{i}-a_{i}\right)^{2}}\right]\left(b_{i}-a_{i}\right) M_{i} .
\end{aligned}
$$

In 1998, Barnett and Dragomir [3] proved the following Ostrowski type inequality for mappings of two variables with bounded second order partial derivatives.

Theorem 3. Let $f:[a, b] \times[c, d] \longrightarrow \mathbb{R}$ continuous on $[a, b] \times$ $[c, d], f_{x, y}^{\prime \prime}=\partial^{2} f / \partial x \partial y$ exists on $(a, b) \times(c, d)$ and is bounded, i.e.,

$$
\left\|f_{s, t}^{\prime \prime}\right\|_{\infty}=\sup _{(x, y) \in(a, b) \times(c, d)}\left|\frac{\partial^{2} f(x, y)}{\partial x \partial y}\right|<\infty,
$$

Then we have the inequality

$$
\begin{aligned}
& \mid \int_{a}^{b} \int_{c}^{d} f(s, t) d s d t-\left[(b-a) \int_{c}^{d} f(x, t) d t\right. \\
& \left.\quad+(d-c) \int_{a}^{b} f(s, y) d s-(d-c)(b-a) f(x, y)\right] \mid \\
& \quad \leq\left[\frac{1}{4}(b-a)^{2}+\left(x-\frac{a+b}{2}\right)^{2}\right]\left[\frac{1}{4}(d-c)^{2}+(y\right. \\
& \left.\left.\quad-\frac{c+d}{2}\right)^{2}\right]\left\|f_{s, t}^{\prime \prime}\right\|_{\infty},
\end{aligned}
$$

for all $(x, y) \in[a, b] \times[c, d]$. 
In [4], Xue et al. derive the following inequality of Ostrowski type.

Theorem 4. Let $f:[a, b] \times[c, d] \longrightarrow \mathbb{R}$ be an absolutely continuous function such that the partial derivatives of order two exist and suppose that there exist constants $\gamma, \Gamma \in \mathbb{R}$ with $\gamma \leq \partial^{2} f(t, s) / \partial t \partial s \leq \Gamma$ for all $(t, s) \in[a, b] \times[c, d]$. Then we have

$$
\begin{aligned}
(1-\lambda)^{2} f(x, y) \\
+\frac{\lambda}{2}(1-\lambda)[f(a, y)+f(b, y)+f(x, c)+f(x, d)] \\
+\left(\frac{\lambda}{2}\right)^{2}[f(a, c)+f(b, c)+f(a, d)+f(b, d)] \\
-\frac{1}{b-a}\left[(1-\lambda) \int_{a}^{b} f(t, y) d t\right. \\
\left.+\frac{\lambda}{2} \int_{a}^{b}[f(t, c)+f(t, d)] d t\right] \\
-\frac{1}{d-c}\left[(1-\lambda) \int_{c}^{d} f(x, s) d s\right. \\
\left.+\frac{\lambda}{2} \int_{c}^{d}[f(a, s)+f(b, s)] d s\right] \\
-\frac{\Gamma+\gamma}{2}(1-\lambda)^{2}\left(x-\frac{a+b}{2}\right)\left(y-\frac{c+d}{2}\right) \\
+\frac{1}{(b-a)(d-c)} \int_{a}^{b} \int_{c}^{d} f(t, s) d s d t \mid \leq \frac{\Gamma-\gamma}{2} \\
+\frac{1}{(b-a)(d-c)}\left[\left(\lambda^{2}+(1-\lambda)^{2}\right) \frac{(b-a)^{2}}{4}\right. \\
\left.+\left(x-\frac{a+b}{2}\right)^{2}\right] \times\left[\left(\lambda^{2}+(1-\lambda)^{2}\right) \frac{(d-c)^{2}}{4}\right. \\
\left.+\left(y-\frac{c+d}{2}\right)^{2}\right],
\end{aligned}
$$

for all $(x, y) \in[a+\lambda((b-a) / 2), b-\lambda((b-a) / 2)] \times[c+$ $\lambda((d-c) / 2), d-\lambda((d-c) / 2)]$ and $\lambda \in[0,1]$.

More recently, Sarikaya et al. [5] establish weighted Ostrowski type inequalities considering function whose second order partial derivatives are bounded as follows.

Theorem 5. Let $f:[a, b] \times[c, d] \longrightarrow \mathbb{R}$ be an absolutely continuous function such that the partial derivatives of order two exist and are bounded, i.e.,

$$
\left\|\frac{\partial^{2} f(t, s)}{\partial t \partial s}\right\|_{\infty}=\sup _{(x, y) \in(a, b) \times(c, d)}\left|\frac{\partial^{2} f(t, s)}{\partial t \partial s}\right|<\infty,
$$

for all $(t, s) \in[a, b] \times[c, d]$. Then we have

$$
\begin{aligned}
& \mid m_{\circ}(a, b) m_{\circ}(c, d)(x-\mu(a, b))(y-\mu(c, d)) f(x, y) \\
& -m_{\circ}(c, d)(y-\mu(c, d))\left[\int_{a}^{x}\left(\int_{a}^{t} \omega(u) d u\right) f(t, y) d t\right. \\
& \left.+\int_{x}^{b}\left(\int_{b}^{t} \omega(u) d u\right) f(t, y) d t\right]-m_{\circ}(a, b)(x \\
& -\mu(a, b))\left[\int_{c}^{y}\left(\int_{c}^{s} \omega(v) d v\right) f(x, s) d s\right. \\
& \left.+\int_{y}^{d}\left(\int_{d}^{s} \omega(v) d v\right) f(x, s) d s\right]-m_{\circ}(a, b) m_{\circ}(c, d) \\
& \cdot \int_{a}^{b} \int_{c}^{d} f(t, s) d s d t \mid \leq \frac{m_{\circ}(a, b) m_{\circ}(c, d)}{4}[(x \\
& \left.-\mu(a, b))^{2}+\sigma^{2}(a, b)\right]\left[(y-\mu(c, d))^{2}+\sigma^{2}(c, d)\right] \\
& .\left\|\frac{\partial^{2} f(t, s)}{\partial t \partial s}\right\|_{\infty} \leq \frac{m_{\circ}(a, b) m_{\circ}(c, d)}{4}\left(\left|x-\frac{a+b}{2}\right|\right. \\
& \left.+\frac{b-a}{2}\right)^{2}\left(\left|y-\frac{c+d}{2}\right|+\frac{d-c}{2}\right)^{2}\left\|\frac{\partial^{2} f(t, s)}{\partial t \partial s}\right\|_{\infty},
\end{aligned}
$$

where

$$
\begin{aligned}
m_{i}(a, b) & =\int_{a}^{b} t^{i} \omega(t) d t, \quad i=0,1, \ldots, \\
\mu(a, b) & =\frac{m_{1}(a, b)}{m_{\circ}(a, b)} \\
\sigma^{2}(a, b) & =\frac{m_{2}(a, b)}{m_{\circ}(a, b)}-\mu^{2}(a, b) .
\end{aligned}
$$

For other related work, we refer the reader to [6-15].

In this paper, motivated by the ideas in both $[4,5]$, we shall derive a new inequality of Ostrowski's type similar to the inequalities (5) and (7), involving functions of two independent variables.

\section{Main Results}

In order to introduce our main results, we commence with the following lemma.

Lemma 6. Let $f:[a, b] \times[c, d] \longrightarrow \mathbb{R}$ be an absolutely continuous function such that the partial derivative of order 4 exists for all $(x, y) \in[a+h((b-a) / 2), b-h((b-a) / 2)] \times$ $[c+h((d-c) / 2), d-h((d-c) / 2)]$ and $h \in[0,1]$. Then for any two mappings $K_{1}(t ; x):[a, b] \times[a, b] \longrightarrow \mathbb{R}$ and $K_{2}(s ; y):[c, d] \times[c, d] \longrightarrow \mathbb{R}$, where

$$
K_{1}(t ; x):= \begin{cases}\frac{1}{2}\left[t-\left(a+h \frac{b-a}{2}\right)\right]^{2}, & t \in[a, x] \\ \frac{1}{2}\left[t-\left(b-h \frac{b-a}{2}\right)\right]^{2}, & t \in(x, b]\end{cases}
$$


and

$$
K_{2}(s ; y):= \begin{cases}\frac{1}{2}\left[s-\left(c+h \frac{d-c}{2}\right)\right]^{2}, & t \in[c, y] \\ \frac{1}{2}\left[s-\left(d-h \frac{d-c}{2}\right)\right]^{2}, & t \in(y, d],\end{cases}
$$

the identity

$$
\begin{aligned}
& E(f ; h)=\int_{a}^{b} \int_{c}^{d} K_{1}(t ; x) K_{2}(s ; y) \frac{\partial^{4} f(t, s)}{\partial t^{2} \partial s^{2}} d s d t=(1 \\
& -h)^{2}(b-a)(d-c)\left[f(x, y)+\left(\frac{a+b}{2}-x\right)\right. \\
& \cdot\left(\frac{c+d}{2}-y\right) f_{t s}(x, y)+\left(\frac{a+b}{2}-x\right) f_{t}(x, y) \\
& \left.+\left(\frac{c+d}{2}-y\right) f_{s}(x, y)\right]+h^{2}(1-h) \\
& \frac{(b-a)(d-c)}{8}\left[(d-c)\left(\frac{a+b}{2}-x\right)\right. \\
& \cdot\left(f_{t s}(x, c)-f_{t s}(x, d)\right)+(b-a)\left(\frac{c+d}{2}-y\right) \\
& \left.\cdot\left(f_{t s}(a, y)-f_{t s}(b, y)\right)\right]+h^{4} \\
& \frac{(b-a)^{2}(d-c)^{2}}{64}\left[f_{t s}(a, c)-f_{t s}(a, d)-f_{t s}(b, c)\right. \\
& \left.+f_{t s}(b, d)\right]+h^{2} \frac{(b-a)^{2}}{8}\{(1-h)(d-c) \\
& \cdot\left[f_{t}(a, y)-f_{t}(b, y)\right] \\
& +h \frac{d-c}{2}\left[f_{t}(a, c)+f_{t}(b, c)+f_{t}(a, d)-f_{t}(b, d)\right] \\
& \left.-\int_{c}^{d}\left[f_{t}(a, s)-f_{t}(b, s)\right] d s\right\}+\frac{h^{2}(d-c)^{2}}{8}\{(1-h) \\
& \cdot(b-a)\left[f_{s}(x, c)-f_{s}(x, d)\right]+h \\
& \text {. } \frac{b-a}{2}\left[f_{s}(a, c)+f_{s}(b, c)+f_{s}(a, d)-f_{s}(b, d)\right] \\
& \left.-\int_{a}^{b}\left[f_{s}(t, c)-f_{s}(t, d)\right] d t\right\}+(1-h)(d-c)\left(\frac{c+d}{2}\right. \\
& -y)\left[h \frac{b-a}{2}\left[f_{s}(a, y)+f_{s}(b, y)\right]-\int_{a}^{b} f_{s}(t, y) d t\right] \\
& +(1-h)(b-a)\left(\frac{a+b}{2}-x\right) \\
& \cdot\left[h \frac{d-c}{2}\left[f_{t}(x, c)+f_{t}(x, d)\right]\right. \\
& \left.-\int_{c}^{d} f_{t}(x, s) d s\right]+h(1-h) \frac{(b-a)(d-c)}{2}[f(a, y) \\
& +f(b, y)+f(x, c)+f(x, d)]+h^{2}
\end{aligned}
$$

$$
\begin{aligned}
& \cdot \frac{(b-a)(d-c)}{4}[f(a, c) \\
& +f(b, c)+f(a, d)+f(b, d)]-(1-h)[(d-c) \\
& \left.\cdot \int_{a}^{b} f(t, y) d t+(b-a) \int_{c}^{d} f(x, s) d s\right]-\frac{h}{2}[(d-c) \\
& \cdot \int_{a}^{b}[f(t, c)+f(t, d)] d t+(b-a) \\
& \left.\cdot \int_{c}^{d}[f(a, s)+f(b, s)] d s\right]+\int_{a}^{b} \int_{c}^{d} f(t, s) d s d t .
\end{aligned}
$$

holds.

Proof. By definitions of $K_{1}(t ; x)$ and $K_{2}(s ; y)$ in both $(9)$ and (10), we have

$$
\begin{gathered}
E(f ; h)=\int_{a}^{b} \int_{c}^{d} K_{1}(t ; x) K_{2}(s ; y) \frac{\partial^{4} f(t, s)}{\partial t^{2} \partial s^{2}} d s d t=\frac{1}{4} \\
\cdot \int_{a}^{x} \int_{c}^{y}\left[t-\left(a+h \frac{b-a}{2}\right)\right]^{2} \\
\cdot\left[s-\left(c+h \frac{d-c}{2}\right)\right]^{2} \frac{\partial^{4} f(t, s)}{\partial t^{2} \partial s^{2}} d s d t \\
+\frac{1}{4} \int_{a}^{x} \int_{y}^{d}\left[t-\left(a+h \frac{b-a}{2}\right)\right]^{2} \\
\cdot\left[s-\left(d-h \frac{d-c}{2}\right)\right]^{2} \frac{\partial^{4} f(t, s)}{\partial t^{2} \partial s^{2}} d s d t \\
+\frac{1}{4} \int_{x}^{b} \int_{c}^{y}\left[t-\left(b-h \frac{b-a}{2}\right)\right]^{2} \\
\cdot\left[s-\left(c+h \frac{d-c}{2}\right)\right]^{2} \frac{\partial^{4} f(t, s)}{\partial t^{2} \partial s^{2}} d s d t \\
+\frac{1}{4} \int_{x}^{b} \int_{y}^{d}\left[t-\left(b-h \frac{b-a}{2}\right)\right]^{2} \\
+\left[s-\left(d-h \frac{d-c}{2}\right)\right]^{2} \frac{\partial^{4} f(t, s)}{\partial t^{2} \partial s^{2}} d s d t \\
\quad=I_{1}+I_{2}+I_{3}+I_{4} .
\end{gathered}
$$

For $I_{1}$, integration by parts yields

$$
\begin{aligned}
I_{1} & =\frac{1}{4} \int_{a}^{x} \int_{c}^{y}\left[t-\left(a+h \frac{b-a}{2}\right)\right]^{2} \\
& \cdot\left[s-\left(c+h \frac{d-c}{2}\right)\right]^{2} \frac{\partial^{4} f(t, s)}{\partial t^{2} \partial s^{2}} d s d t \\
& =\frac{1}{4}\left[t-\left(a+h \frac{b-a}{2}\right)\right]^{2}\left[s-\left(c+h \frac{d-c}{2}\right)\right]^{2} \\
& \cdot f_{t s}(x, y)-\frac{h^{2}}{16}\left[(d-c)^{2}\left[x-\left(a+h \frac{b-a}{2}\right)\right]^{2}\right.
\end{aligned}
$$




$$
\begin{aligned}
& \left.\cdot f_{t s}(x, c)+(b-a)^{2}\left[y-\left(c+h \frac{d-c}{2}\right)\right]^{2} f_{t s}(a, y)\right] \\
& +\frac{h^{4}(b-a)^{2}(d-c)^{2}}{64} f_{t s}(a, c)+\frac{h^{2}(d-c)^{2}}{8} \int_{x}^{b}[t \\
& \left.-\left(b-h \frac{b-a}{2}\right)\right] f_{t s}(t, c) d t-\frac{h^{2}(b-a)^{2}}{8} \int_{c}^{y}[s \\
& \left.-\left(c+h \frac{d-c}{2}\right)\right] f_{t s}(b, s) d s+\frac{1}{2} \int_{c}^{y}[s \\
& \left.-\left(c+h \frac{d-c}{2}\right)\right]\left[x-\left(b-h \frac{b-a}{2}\right)\right]^{2} f_{t s}(x, s) d s \\
& -\frac{1}{2} \int_{x}^{b}\left[t-\left(b-h \frac{b-a}{2}\right)\right]\left[y-\left(c+h \frac{d-c}{2}\right)\right]^{2} \\
& \cdot f_{t s}(t, y) d t+\int_{a}^{x} \int_{c}^{y}\left[t-\left(a+h \frac{b-a}{2}\right)\right] \\
& \cdot\left[s-\left(c+h \frac{d-c}{2}\right)\right] f_{t s}(t, s) d s d t .
\end{aligned}
$$

Similarly, $I_{2}, I_{3}$, and $I_{4}$ can be obtained.

Thus, by adding $I_{1}, I_{2}, I_{3}$, and $I_{4}$, we easily deduce

$$
\begin{aligned}
& E(f ; h)=\int_{a}^{b} \int_{c}^{d} K_{1}(t ; x) K_{2}(s ; y) \frac{\partial^{4} f(t, s)}{\partial t^{2} \partial s^{2}} d s d t=\frac{1}{4}[(h \\
& -1)(b-a)(a+b-2 x)][(h-1)(d-c)(c+d-2 y)] \\
& \cdot f_{t s}(x, y)-\frac{h^{2}}{16}(d-c)^{2}(h-1)(b-a)(a+b-2 x) \\
& \cdot\left[f_{t s}(x, c)-f_{t s}(x, d)\right]-\frac{h^{2}}{16}(b-a)^{2}(h-1)(d-c)(c \\
& +d-2 y)\left[f_{t s}(a, y)-f_{t s}(b, y)\right] \\
& +\frac{h^{4}(b-a)^{2}(d-c)^{2}}{64}\left[f_{t s}(a, c)-f_{t s}(a, d)-f_{t s}(b, c)\right. \\
& +f(b, d)]+\frac{h^{2}(b-a)^{2}}{8}\left[\int_{c}^{y}\left[s-\left(c+h \frac{d-c}{2}\right)\right]\right. \\
& \cdot\left[f_{t s}(a, s)-f_{t s}(b, s)\right] d s+\int_{y}^{d}\left[s-\left(d-h \frac{d-c}{2}\right)\right] \\
& \left.\cdot\left[f_{t s}(a, s)-f_{t s}(b, s)\right] d s\right] \\
& +\int_{a}^{x} \int_{c}^{y}\left[t-\left(a+h \frac{b-a}{2}\right)\right]\left[s-\left(c+h \frac{d-c}{2}\right)\right] \\
& f_{t s}(t, s) d s d t+\int_{a}^{x} \int_{y}^{d}\left[t-\left(a+h \frac{b-a}{2}\right)\right] \\
& \cdot\left[s-\left(d-h \frac{d-c}{2}\right)\right] f_{t s}(t, s) d s d t \\
& +\int_{x}^{b} \int_{c}^{y}\left[t-\left(b-h \frac{b-a}{2}\right)\right]\left[s-\left(c+h \frac{d-c}{2}\right)\right]
\end{aligned}
$$

$$
\begin{aligned}
& \cdot f_{t s}(t, s) d s d t+\int_{x}^{b} \int_{y}^{d}\left[t-\left(b-h \frac{b-a}{2}\right)\right] \\
& \cdot\left[s-\left(d-h \frac{d-c}{2}\right)\right] f_{t s}(t, s) d s d t .
\end{aligned}
$$

By further algebraic manipulations and assuming result by [4], the proof of Lemma 6 is completed.

Theorem 7. Let $f:[a, b] \times[c, d] \longrightarrow \mathbb{R}$ such that $f \in$ $C^{4}([a, b] \times[c, d])$ be an absolutely continuous function such that the partial derivative of order 4 exists and is bounded; i.e.,

$$
\left\|\frac{\partial^{4} f(t, s)}{\partial t^{2} \partial s^{2}}\right\|_{\infty}=\sup _{(x, y) \in(a, b) \times(c, d)}\left|\frac{\partial^{4} f(t, s)}{\partial t^{2} \partial s^{2}}\right|<\infty,
$$

for all $(t, s) \in[a, b] \times[c, d]$. Then for all $(x, y) \in[a+h((b-$

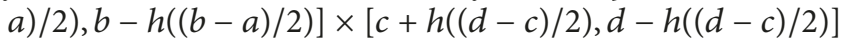
and $h \in[0,1]$, we have

$$
\begin{aligned}
\mid E & (f ; h) \mid \leq\left[\frac{h^{3}(b-a)^{3}}{24}\right. \\
& +\frac{(1-h)(b-a)}{2}\left(\frac{(1-h)^{2}(b-a)^{2}}{12}\right. \\
& \left.\left.+\left(x-\frac{a+b}{2}\right)^{2}\right)\right] \times\left[\frac{h^{3}(d-c)^{3}}{24}\right. \\
& +\frac{(1-h)(d-c)}{2}\left(\frac{(1-h)^{2}(d-c)^{2}}{12}\right. \\
& \left.\left.+\left(y-\frac{c+d}{2}\right)^{2}\right)\right]\left\|\frac{\partial^{4} f(t, s)}{\partial t^{2} \partial s^{2}}\right\|_{\infty},
\end{aligned}
$$

where the functional $E(f ; h)$ is given by (11).

Proof. By considering (11), we have

$$
\begin{aligned}
|E(f ; h)| & =\left|\int_{a}^{b} \int_{c}^{d} K_{1}(t ; x) K_{2}(s ; y) \frac{\partial^{4} f(t, s)}{\partial t^{2} \partial s^{2}} d s d t\right| \\
& \leq \int_{a}^{b} \int_{c}^{d}\left|K_{1}(t ; x)\right|\left|K_{2}(s ; y)\right| \frac{\partial^{4} f(t, s)}{\partial t^{2} \partial s^{2}} \mid d s d t \\
& \leq\left\|\frac{\partial^{4} f(t, s)}{\partial t^{2} \partial s^{2}}\right\|_{\infty} \int_{a}^{b} K_{1}(t ; x) d t \cdot \int_{c}^{d} K_{2}(s ; y) d s .
\end{aligned}
$$

But,

$$
\begin{aligned}
& \int_{a}^{b} K_{1}(t ; x) d t=\frac{h^{3}}{24}(b-a)^{3} \\
& \quad+\frac{(1-h)(b-a)}{2}\left(\frac{(1-h)^{2}(b-a)^{2}}{12}+\left[x-\frac{a+b}{2}\right]^{2}\right),
\end{aligned}
$$

and

$$
\begin{aligned}
& \int_{c}^{d} K_{2}(s ; y) d s=\frac{h^{3}}{24}(d-c)^{3} \\
& \quad+\frac{(1-h)(d-c)}{2}\left(\frac{(1-h)^{2}(d-c)^{2}}{12}+\left[y-\frac{c+d}{2}\right]^{2}\right) .
\end{aligned}
$$


Now, substituting (18), (19) into (17) gives (16) and, hence, completes the proof.

Corollary 8. Under the assumption of Theorem 7 with $h=0$, we have

$$
\begin{aligned}
& \mid f(x, y)+\left(\frac{a+b}{2}-x\right)\left(\frac{c+d}{2}-y\right) f_{t s}(x, y) \\
& +\left(\frac{a+b}{2}-x\right) f_{t}(x, y)+\left(\frac{c+d}{2}-y\right) f_{s}(x, y) \\
& -\left[\frac{1}{(b-a)}\left(\frac{c+d}{2}-y\right) \int_{a}^{b} f_{s}(t, y) d t\right. \\
& \left.+\frac{1}{(d-c)}\left(\frac{a+b}{2}-x\right) \int_{c}^{d} f_{t}(x, s) d s\right] \\
& -\left[\frac{1}{(b-a)} \int_{a}^{b} f(t, y) d t+\frac{1}{(d-c)} \int_{c}^{d} f(x, s) d s\right] \\
& +\frac{1}{(b-a)(d-c)} \int_{a}^{b} \int_{c}^{d} f(t, s) d s d t \mid \leq \frac{1}{4}\left(\frac{(b-a)^{2}}{12}\right. \\
& \left.+\left(x-\frac{a+b}{2}\right)^{2}\right)\left(\frac{(d-c)^{2}}{12}+\left(y-\frac{c+d}{2}\right)^{2}\right) \\
& +\left\|\frac{\partial^{4} f(t, s)}{\partial t^{2} \partial s^{2}}\right\|_{\infty} \cdot
\end{aligned}
$$

Corollary 9. Under the assumption of Theorem 7 with $h=0$, $x=(a+b) / 2$, and $y=(c+d) / 2$ we have

$$
\begin{aligned}
& \mid f\left(\frac{a+b}{2}, \frac{c+d}{2}\right)-\left[\frac{1}{(b-a)} \int_{a}^{b} f\left(t, \frac{c+d}{2}\right) d t\right. \\
& \left.\quad+\frac{1}{(d-c)} \int_{c}^{d} f\left(\frac{a+b}{2}, s\right) d s\right]+\frac{1}{(b-a)(d-c)} \\
& \quad \cdot \int_{a}^{b} \int_{c}^{d} f(t, s) d s d t \mid \\
& \quad \leq \frac{(b-a)^{2}(d-c)^{2}}{576}\left\|\frac{\partial^{4} f(t, s)}{\partial t^{2} \partial s^{2}}\right\|_{\infty} .
\end{aligned}
$$

Corollary 10. Under the assumption of Theorem 7 with $h=0$, $x=(a+b) / 4$, and $y=(c+d) / 4$ we have

$$
\begin{aligned}
& \mid f\left(\frac{a+b}{4}, \frac{c+d}{4}\right)+\frac{(a+b)(c+d)}{16} f_{t s}\left(\frac{a+b}{4}, \frac{c+d}{4}\right) \\
& +\left(\frac{a+b}{4}\right) f_{t}\left(\frac{a+b}{4}, \frac{c+d}{4}\right)+\left(\frac{c+d}{4}\right) \\
& \cdot f_{s}\left(\frac{a+b}{4}, \frac{c+d}{4}\right)-\frac{1}{4}\left[\frac{(d-c)}{(b-a)} \int_{a}^{b} f_{s}\left(t, \frac{c+d}{4}\right) d t\right. \\
& \left.+\frac{(b-a)}{(d-c)} \int_{c}^{d} f_{t}\left(\frac{a+b}{4}, s\right) d s\right]
\end{aligned}
$$

$$
\begin{aligned}
& -\left[\frac{1}{(b-a)} \int_{a}^{b} f\left(t, \frac{c+d}{4}\right) d t\right. \\
& \left.+\frac{1}{(d-c)} \int_{c}^{d} f\left(\frac{a+b}{4}, s\right) d s\right] \\
& +\frac{1}{(b-a)(d-c)} \int_{a}^{b} \int_{c}^{d} f(t, s) d s d t \mid \leq \frac{1}{4}\left[\frac{(b-a)^{2}}{12}\right. \\
& \left.+\frac{(a+b)^{2}}{16}\right]\left[\frac{(d-c)^{2}}{12}+\frac{(c+d)^{2}}{16}\right]\left\|\frac{\partial^{4} f(t, s)}{\partial t^{2} \partial s^{2}}\right\|_{\infty} .
\end{aligned}
$$

Remark 11. In Corollaries 8, 9, and 10 we assume that the involved integrals can more easily be computed than the original double integral.

\section{Data Availability}

No data were used to support this study.

\section{Conflicts of Interest}

The author declares no conflicts of interest.

\section{References}

[1] A. Ostrowski, "Über die Absolutabweichung einer differentiebaren Funktion von ihrem Integralmittelwert," Commentarii Mathematici Helvetici, vol. 10, no. 1, pp. 226-227, 1937.

[2] G. V. Milovanovic, On Some Integral Inequalities, Univ. Beograd. Publ. Elektrotehn. Fak. Ser. Math. Fiz., (498/541), pp, 119-124, 1975.

[3] N. S. Barnett and S. S. Dragomir, "An Ostrowski type inequality for double integrals and applications for cubature formulae," Soochow Journal of Mathematics, vol. 27, no. 1, pp. 1-10, 2001.

[4] Q. Xue, J. Zhu, and W. Liu, "A new generalization of Ostrowskitype inequality involving functions of two independent variables," Computers \& Mathematics with Applications, vol. 60, no. 8, pp. 2219-2224, 2010.

[5] M. Z. Sarikaya, H. Yaldiz, and S. Erden, "On the weighted Ostrowski type inequalities for double integrals," Kragujevac Journal of Mathematics, vol. 38, no. 2, pp. 303-314, 2014.

[6] W. G. Alshanti and A. Qayyum, "A note on new Ostrowski type inequalities using a generalized kernel," Bulletin of Mathematical Analysis and Applications, vol. 9, no. 1, pp. 74-91, 2017.

[7] W. G. Alshanti, A. Qayyum, and M. A. Majid, "Ostrowski type inequalities by using generalized quadratic kernel," Journal of Inequalities and Special Functions, vol. 8, no. 4, pp. 111-135, 2017.

[8] W. G. Alshanti, "A Perturbed Version of General Weighted Ostrowski Type Inequality and Applications," International Journal of Analysis and Applications, vol. 16, no. 4, pp. 503-517, 2018.

[9] S. Erden, H. Budak, and M. Z. Sarikaya, "An Ostrowski type inequality for twice differentiable mappings and applications," Mathematical Modelling and Analysis, vol. 21, no. 4, pp. 522-532, 2016.

[10] S. S. Dragomir, P. Cerone, N. S. Barnett, and J. Roumeliotis, "An inequality of the Ostrowski type for double integrals and 
applications for cubature formulae," RGMIA Research Report Collection, vol. 2, no. 6, pp. 1-13, 1999.

[11] B. G. Pachpatte, "On an inequality of Ostrowski type in three independent variables," Journal of Mathematical Analysis and Applications, vol. 249, no. 2, pp. 583-591, 2000.

[12] B. G. Pachpatte, "On multivariate Ostrowski type inequalities," Journal of Inequalities in Pure and Applied Mathematics, vol. 3, no. 4, pp. 1-12, 2002.

[13] M. Z. Sarikaya, "On the Ostrowski type integral inequality for double integrals," Demonstratio Mathematica, vol. 45, no. 3, pp. 533-540, 2012.

[14] M. Z. Sarikaya and H. Ogunmez, "On the weighted Ostrowskitype integral inequality for double integrals," Arabian Journal for Science and Engineering, vol. 36, no. 6, pp. 1153-1160, 2011.

[15] G. Farid, "New Ostrowski-type inequalities and their applications in two coordinates," Acta Mathematica Universitatis Comenianae, vol. 85, no. 1, pp. 107-112, 2016. 


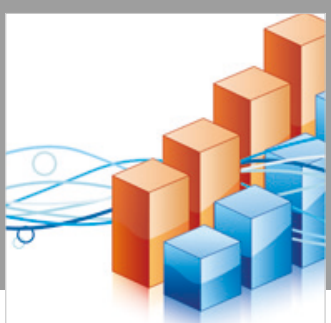

Advances in

Operations Research

\section{-n-m}
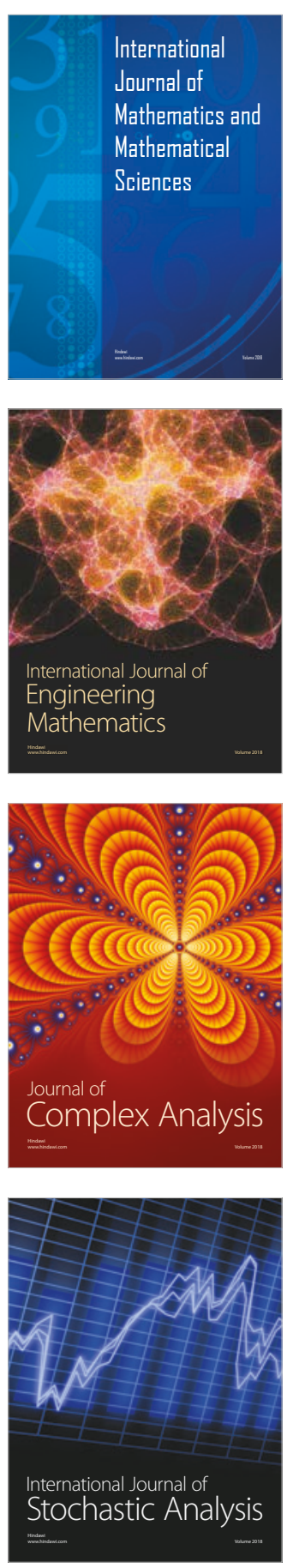
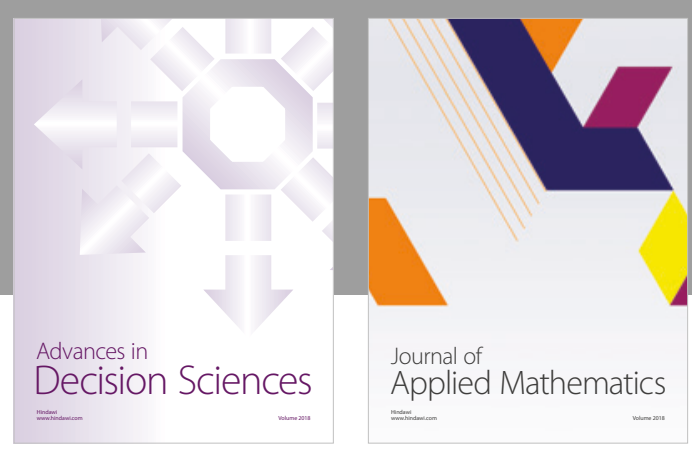

Journal of

Applied Mathematics
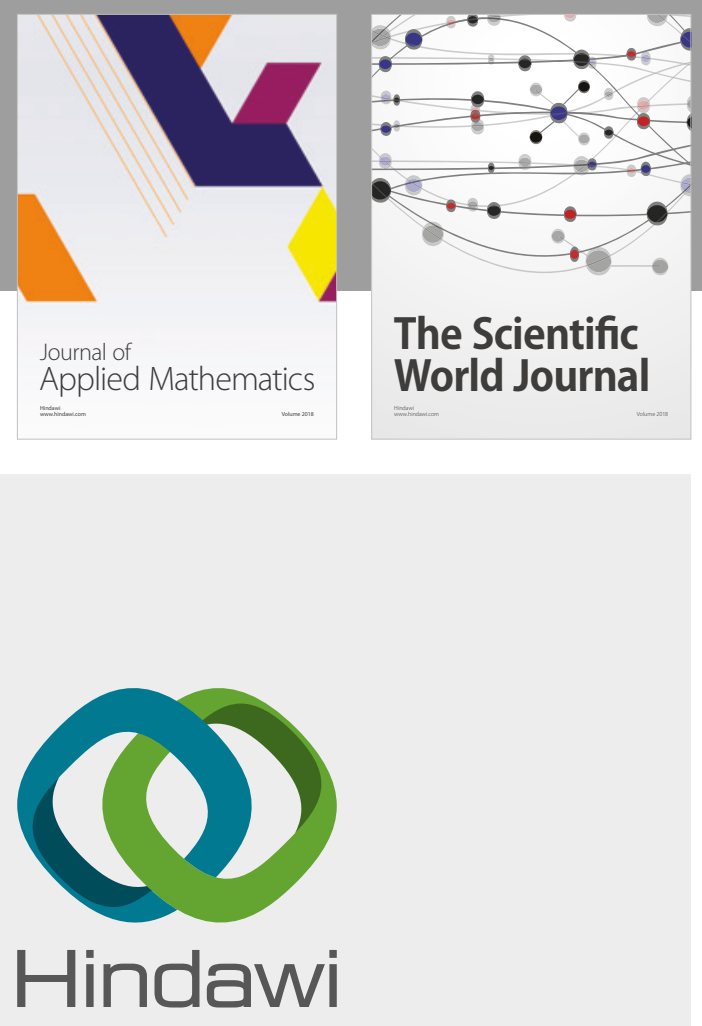

Submit your manuscripts at

www.hindawi.com

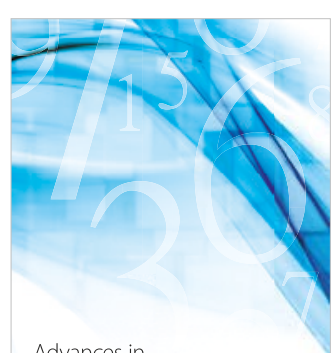

Advances in
Numerical Analysis
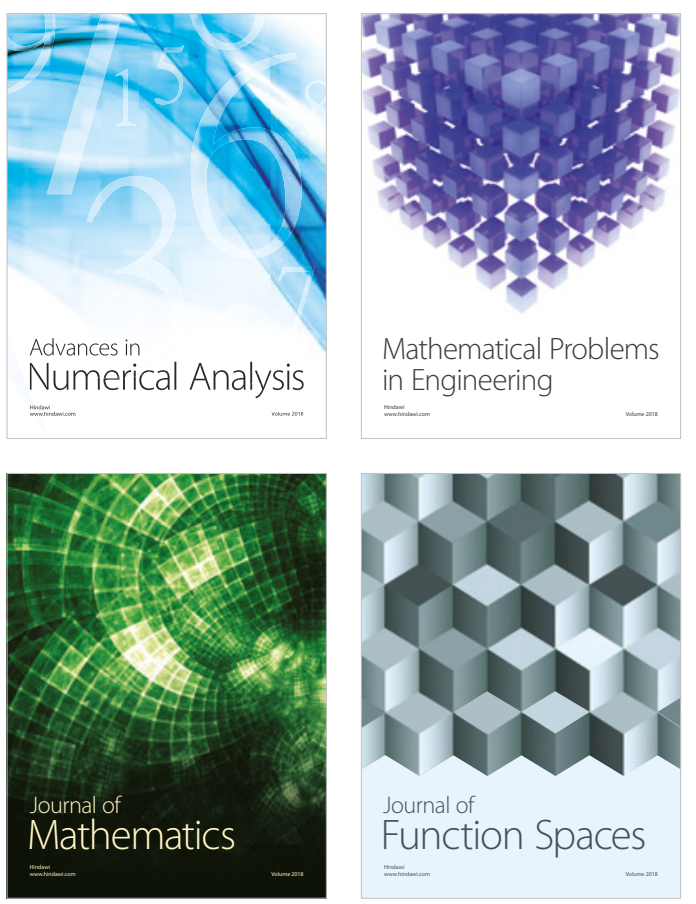

Mathematical Problems in Engineering

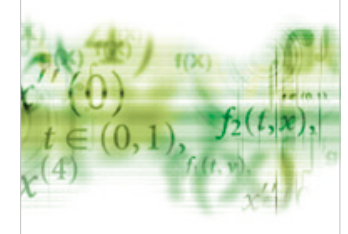

International Journal of

Differential Equations

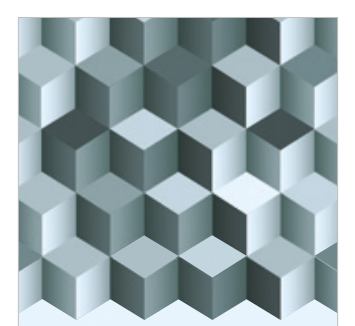

Journal of

Function Spaces

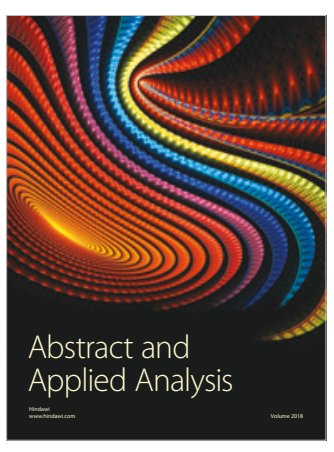

The Scientific

World Journal

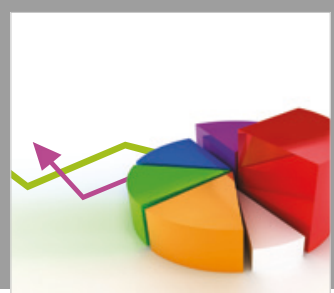

Journal of

Probability and Statistics
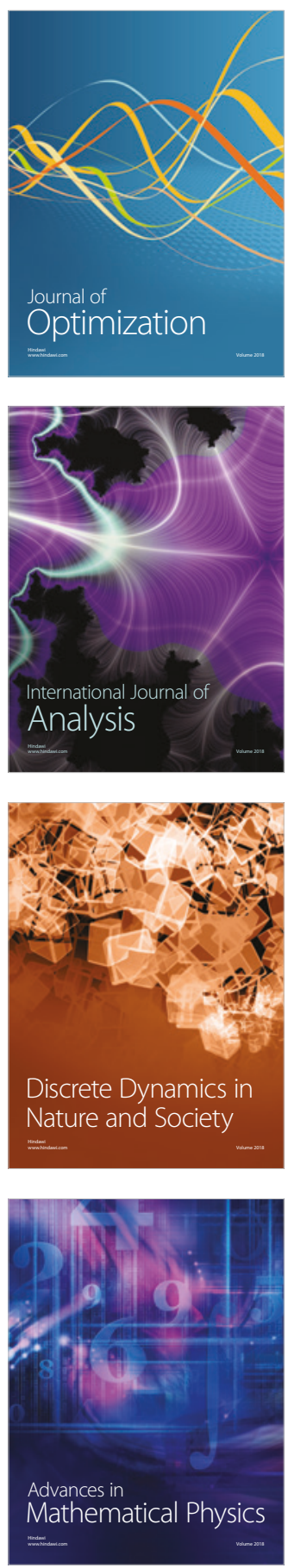